\title{
Chromatic number of sparse colored mixed planar graphs
}

\author{
Amanda Montejano $^{\mathrm{a}, 1}$ Alexandre Pinlou ${ }^{\mathrm{b}, 1}$ André Raspaud ${ }^{\mathrm{c}, 1}$ \\ Éric Sopena ${ }^{c, 1}$ \\ ${ }^{a}$ Dept. Matemàtica Aplicada IV, UPC, Barcelona, Spain \\ b LIRMM, Univ. Montpellier 2, 161 rue Ada, 34392 Montpellier Cedex 5, France \\ ${ }^{\mathrm{c}}$ LaBRI, Univ. Bordeaux 1, 351 Cours de la Libération, 33405 Talence, France
}

\begin{abstract}
A colored mixed graph has vertices linked by both colored arcs and colored edges. The chromatic number of such a graph $G$ is defined as the smallest order of a colored mixed graph $H$ such that there exists a (arc-color preserving) homomorphism from $G$ to $H$. We study in this paper the colored mixed chromatic number of planar graphs, partial 2-trees and outerplanar graphs with given girth.
\end{abstract}

Keywords: mixed graph, planar, girth, chromatic number, homomorphism.

\section{Introduction}

Our general aim is to study homomorphisms of $(n, m)$-colored mixed graphs, which are graphs with both arcs and edges colored with $n$ and $m$ colors, respectively. This notion was introduced by Nešetřil and Raspaud [5] as a common generalization of the notion of edge-colored graphs, obtained by taking $(n, m)=(0, m)$, and the

$\overline{1}$ Emails: amanda@ma4.upc.edu, pinlou@lirmm.fr, \{raspaud, sopena\}@labri.fr 
notion of oriented colorings, which arises when $(n, m)=(1,0)$, (see e.g. [1] and [7] respectively).

A mixed graph is obtained from a simple graph, by assigning to some edges one of the two possible orientations; hence, a mixed graph $G$ is usually denoted by an ordered triple $G=(V, A, E)$. An $(n, m)$-colored mixed graph is a mixed graph $G=(V, A, E)$, together with partitions $A=A_{1} \cup \cdots \cup A_{n}$ and $E=E_{1} \cup \cdots \cup E_{m}$ where $A_{i}$ (resp. $E_{i}$ ) consists of the set of arcs (resp. edges) colored by color $i$. Hence, a $(1,1)$-colored mixed graph is just a mixed graph, a $(0,1)$-colored mixed graph is a simple graph, and a $(1,0)$-colored mixed graph is an oriented graph.

Let $G$ and $H$ be two $(n, m)$-colored mixed graphs. A colored homomorphism from $G$ to $H$ is a mapping $h: V(G) \rightarrow V(H)$ that maps edges to edges and arcs to arcs preserving the orientation and the colors, i.e. $(h(u), h(v)) \in A_{i}(H)$ whenever $(u, v) \in A_{i}(G)$ (for every $i \in\{1, \ldots, n\}$ ), and $h(u) h(v) \in E_{j}(H)$ whenever $u v \in E_{j}(G)$ (for every $j \in\{1, \ldots, m\}$ ).

We say that $G$ is $H$-colorable if there exists a colored homomorphism from $G$ to $H$; hence, the vertices of $H$ will be called the colors. For a colored mixed graph $G$, the smallest number of vertices of a colored mixed graph $H$ such that $G$ is $H$-colorable is the colored mixed chromatic number. For a simple graph $G$, the $(n, m)$-colored mixed chromatic number, denoted by $\chi_{(n, m)}(G)$, is defined as the maximum of the colored mixed chromatic numbers taken over all the possible $(n, m)$-colored mixed graphs having $G$ as underlying undirected graph. Note that $\chi_{(0,1)}(G)$ is the ordinary chromatic number, and $\chi_{(1,0)}(G)$ is the oriented chromatic number. Given a (finite or infinite) family of simple graphs $\mathcal{F}$, the $(n, m)$-colored mixed chromatic number of $\mathcal{F}$, denoted by $\chi_{(n, m)}(\mathcal{F})$, is the maximum of $\chi_{(n, m)}(G)$ taken over all members in $\mathcal{F}$.

The most natural question to consider in this framework is whether or not a given family of graphs has finite $(n, m)$-colored mixed chromatic number. In this paper we study the $(n, m)$-colored mixed chromatic number of the following three particular classes of graphs (the girth of a graph is the length of a shortest cycle): $\mathcal{P}_{g}$ (planar graphs with girth at least $g$ ), $\mathcal{T}_{g}^{2}$ (partial 2-trees with girth at least $g$ ), and $O_{g}$ (outerplanar graphs with girth at least $g$ ). Let us denote $\mathcal{P}=\mathcal{P}_{3}, \mathcal{T}^{2}=\mathcal{T}_{3}^{2}$ and $\left.\mathrm{O}=\mathrm{O}_{3}\right)$.

Theorem 1.1 [2,4,5]

(i) $(2 n+m)^{3}+\varepsilon(2 n+m)^{2}+(2 n+m)+\varepsilon \leq \chi_{(n, m)}(\mathcal{P}) \leq 5(2 n+m)^{4}$

(ii) $(2 n+m)^{2}+\varepsilon(2 n+m)+1 \leq \chi_{(n, m)}\left(\mathcal{T}^{2}\right) \leq 3(2 n+m)^{2}$

(iii) $(2 n+m)^{2}+\varepsilon(2 n+m)+1 \leq \chi_{(n, m)}(O) \leq 3(2 n+m)^{2}$

where $\varepsilon=1$ for $m$ odd or $m=0$, and $\varepsilon=2$ for $m>0$ even. 
Observe that, for the class of planar graphs, the main term in the lower bound is $(2 n+m)^{3}$ while the main term in the upper bound is $(2 n+m)^{4}$; concerning partial 2 -trees and outerplanar graphs, the main term in both lower and upper bounds is $(2 n+m)^{2}$.

Motivated by the 4-color Theorem (simple planar graphs are 4-colorable) and Grötzsch Theorem (simple triangle-free planar graphs are 3-colorable), Nešetřil, Raspaud and Sopena [6] studied the same kind of problem in the oriented case $((1,0)$-colored mixed graphs), i.e. they bound the oriented chromatic number of oriented planar graphs with given girth.

Concerning the class of $(0,2)$-colored mixed graphs, the authors in [4] recently bounded the $(0,2)$-colored mixed chromatic number of $\mathcal{P}_{g}, \mathcal{T}_{g}^{2}$ and $O_{g}$.

In this paper, we studied the $(n, m)$-colored mixed chromatic number of planar graph, partial 2-trees and outerplanar graphs with given girth and we prove that, if the girth is sufficiently large, the colored mixed chromatic number becomes linear in $2 n+m$.

\section{Theorem 1.2}

(i) $\chi_{(n, m)}\left(\mathscr{P}_{g}\right)=2(2 n+m)+1$ for every $g \geq 20 n+10 m-4$.

(ii) $\chi_{(n, m)}\left(\mathcal{T}_{g}^{2}\right)=2(2 n+m)+1$ for every $g \geq 8 n+4 m-1$.

(iii) $\chi_{(n, m)}\left(O_{g}\right)=2(2 n+m)+1$ for every $g \geq 4 n+2 m+\beta$, where $\beta=0$ if $n=0$, and $\beta=1$ otherwise.

In Section 2, we define the notion of nice graph which is useful to prove our main theorem and we describe the target graphs that will be used to prove the upper bounds of Theorem 1.2. Due to lack of space, we only give the proof of the upper bound of Theorem 1.2(i) in Section 3 and Section 4 is devoted to the proof of the lower bounds by constructing, for every $g \geq 3$, a colored mixed outerplanar graph with girth $g$ and chromatic number $2(2 n+m)+1$.

\section{Nice colored mixed graphs}

Hell et al. [3] introduced the concept of nice digraphs and nice edge colored multigraphs. We adapt this concept to the context of colored mixed graphs.

An $(n, m)$-colored mixed graph $G$ is $k$-nice (resp. $k$-quasi-nice) if for every pair of non-necessarily distinct (resp. necessarily distinct) vertices $v, w \in V(G)$, and for each $(n, m)$-colored mixed path $P_{k}=u_{0}, u_{1}, \ldots, u_{k}$ of length $k$, there exists a colored homomorphism $h$ from $P_{k}$ to $G$ such that $h\left(u_{0}\right)=v$ and $h\left(u_{k}\right)=w$.

To prove Theorem 1.2, we will show that each considered graph class admits a colored homomorphism to an $(n, m)$-colored mixed graph $T$ of order $4 n+2 m+$ 
1: $T$ is a complete $(n, m)$-colored mixed graph, whose underlying graph is the complete graph $K_{4 n+2 m+1}$. We can decompose the edges of $K_{4 n+2 m+1}$ into $2 n+m$ Hamiltonian cycles $C_{1}$ to $C_{2 n+m}$. For $i \in\{1, \ldots, 2 n\}$, the $C_{i}$ 's are circuits colored by color $\lceil i / 2\rceil$; for $i \in\{2 n+1, \ldots, 2 n+m\}, C_{i}$ 's are cycles colored $i-2 n$.

Proposition 2.1 The $(n, m)$-colored mixed graph $T$ is $(4 n+2 m)$-nice, and $(2 m-$ 1)-quasi-nice when $n=0$.

\section{The upper bounds of Theorem 1.2}

We give in this section the general ideas to get the upper bound of Theorem 1.2(i). The two other upper bounds can be obtained using the same techniques.

Proposition 3.1 [6] Every planar graph with girth g, contains either a vertex of degree one or a path of length $\frac{g+4}{5}$ whose internal vertices are of degree 2.

Lemma 3.2 . Every graph $G \in \mathcal{P}_{5 k-4}$ admits a colored homomorphism to a k-nice colored mixed graph.

Proof (Sketch) Let $H$ be a $k$-nice $(n, m)$-colored mixed graph. We prove that every $(n, m)$-colored mixed planar graph with girth at least $5 k-4$ admits a colored homomorphism to $H$. Note that is sufficient to consider the case $g=5 k-4$. Let $G$ be a minimal (with respect to the number of vertices) counter-example. One can prove that $G$ contains neither a 1-vertex, nor a path of length $k$ whose internal vertices are of degree 2. We thus get a contradiction by Proposition 3.1 .

The upper bound of Theorem 1.2(i) is clearly a direct consequence of Proposition 2.1 and Lemma 3.2.

\section{The lower bounds of Theorem 1.2}

Let $G$ be the $(n, m)$-colored mixed outerplanar graph with girth $g$ obtained as follows. We take two copies $P, P^{\prime}$ of each $(n, m)$-colored mixed path $u_{0}, \ldots, u_{\left\lfloor\frac{g}{2}\right\rfloor}$ of length $\left\lfloor\frac{g}{2}\right\rfloor$ (there exist $(2 n+m)^{\left\lfloor\frac{g}{2}\right\rfloor}$ distinct such paths). We identify all the $2(2 n+m)^{\left\lfloor\frac{g}{2}\right\rfloor} u_{0}$-vertices, and connect (with an edge or an arc of an arbitrary color) the two ends $u_{\left\lfloor\frac{g}{2}\right\rfloor}$-vertices of each pair of paths.

Figure 1 shows this outerplanar colored mixed graph for $(n, m)=(1,1)$ and $g=5$.

Lemma $4.1 \chi_{(n, m)}(G)>4 n+2 m$. 


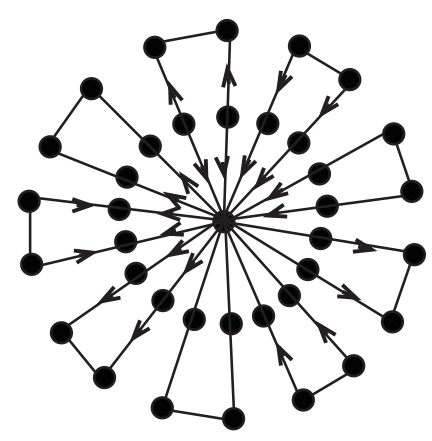

Figure 1. The $(1,1)$-colored mixed outerplanar graph with girth 5 and colored mixed chromatic number 7 .

Proof (Sketch) Let $H$ be any $(n, m)$-colored mixed graph of order $4 n+2 m$. Suppose that there exists a colored homomorphism $h$ from $G$ to $H$ and let $h\left(u_{0}\right)$ be the color of the central vertex of $G$. Since each vertex of $H$ has degree at most $4 n+2 m-1$, one can prove that whatever the color we give to the central vertex of $G$, there exists a pair of $(n, m)$-colored mixed paths of $G$ that is not colorable.

Since $O \subset \mathcal{T}^{2} \subset \mathcal{P}$, this completes the proof of Theorem 1.2.

\section{References}

[1] N. Alon, T.H. Marshall. Homomorphisms of edge-colored graphs and coxeter groups, J. Algebraic Combin. 8 (1998), 5-13.

[2] R. Fabila-Monroy, D. Flores, C. Huemer, A. Montejano. Lower bounds for the colored mixed chromatic number of some clases of graphs, Comment. Math. Univ. Carolin. vol. 49(4) (2008) 637-645.

[3] P. Hell, A. V. Kostochka, A. Raspaud, E. Sopena, On nice graphs, Discrete Math. 234 (2001), 39-51.

[4] A. Montejano, P. Ochem, A. Pinlou, A. Raspaud, E. Sopena. Homomorphisms of $2-$ edge colored graphs, Discrete Applied Mathematics. to appear (2009).

[5] J. Nešetřil, A. Raspaud. Colored homomorphisms of colored mixed graphs, J. Combin. Theory Ser. B 80 (2000), 147-155.

[6] J. Nešetřil, A. Rapaud, E. Sopena. Colorings and girth of oriented planar graphs, Discrete Math. 165/166 (1997), 519-530.

[7] E. Sopena. Oriented graph coloring, Discrete Math. 229 (2001), 359-369. 